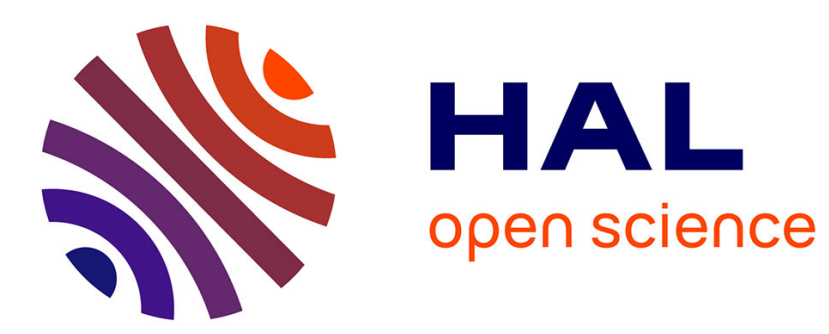

\title{
A comprehensive microscopic model of the extraordinary optical transmission
}

\author{
Haitao Liu, Philippe Lalanne
}

\section{To cite this version:}

Haitao Liu, Philippe Lalanne. A comprehensive microscopic model of the extraordinary optical transmission. Journal of the Optical Society of America. A, Optics and image science, 2010, 27, pp.25422550. hal-00570639

\section{HAL Id: hal-00570639 \\ https://hal-iogs.archives-ouvertes.fr/hal-00570639}

Submitted on 5 Apr 2012

HAL is a multi-disciplinary open access archive for the deposit and dissemination of scientific research documents, whether they are published or not. The documents may come from teaching and research institutions in France or abroad, or from public or private research centers.
L'archive ouverte pluridisciplinaire HAL, est destinée au dépôt et à la diffusion de documents scientifiques de niveau recherche, publiés ou non, émanant des établissements d'enseignement et de recherche français ou étrangers, des laboratoires publics ou privés. 


\title{
Comprehensive microscopic model of the extraordinary optical transmission
}

\author{
Haitao Liu ${ }^{1, *}$ and Philippe Lalanne ${ }^{2}$ \\ ${ }^{1}$ Key Laboratory of Opto-electronic Information Science and Technology, Ministry of Education, \\ Institute of Modern Optics, Nankai University, Tianjin 300071, China \\ ${ }^{2}$ Laboratoire Charles Fabry de l'Institut d'Optique, CNRS, Univ Paris-Sud, Campus Polytechnique, \\ 91127 Palaiseau CEDEX, France \\ *Corresponding author: liuht@nankai.edu.cn
}

Received June 28, 2010; revised September 10, 2010; accepted October 4, 2010; posted October 6, 2010 (Doc. ID 130738); published November 2, 2010

\begin{abstract}
As shown in a recent letter [Nature 452, 728 (2008)] with a microscopic model, the phenomenon of the extraordinary optical transmission (EOT) is intrinsically due to two distinct surface waves: the surface plasmon polariton and the quasi-cylindrical wave (quasi-CW) that efficiently funnel light into the hole aperture at resonance. Here we present a comprehensive microscopic model of the EOT that takes into account the two surface waves. The model preserves the desirable physical insight of the previous approach, but since it additionally takes into account the quasi-CWs, it provides highly accurate predictions over a much broader spectral range, from visible to microwave radiation. The net outcome is a complete understanding of many aspects of the EOT and especially of the role of the metal conductivity that has largely puzzled the initial interpretations. We believe that the main conclusions of the present analysis may be applied to many Wood-type surface resonances on metallic surfaces. (C) 2010 Optical Society of America
\end{abstract}

OCIS codes: $050.1950,050.6624,240.6680$.

\section{INTRODUCTION}

Noble-metal surfaces patterned with sub-wavelength (sub- $\lambda$ ) structures play a central role in the emerging field of plasmonics and metamaterials [1-3]. Although the representation of a multiple-scattering progression of surface plasmon polariton (SPP) waves, initially launched by some indentations and successively scattered by neighboring indentations, is widely accepted and conceptually attractive to comprehend the physics of the surface [4-13], it is scarcely used in the earlier stages of the design. One often prefers to perform fully vectorial calculations [14-16], which when feasible, provide important information like the spatial and temporal field distributions. However, because the numerical approach is by essence different from our physical representation, it is often difficult to recover the initial SPP-progression picture from the calculated data. Thus, even for basic metallo-dielectric geometries such as gratings, one usually ignores how much SPPs are excited on the flat parts of the nano-structured surface in between the indentations and how these SPPs participate into the optical response [17]. Feedback from the numerical solution to the intuitive picture is missing.

The extraordinary optical transmission (EOT) through a sub- $\lambda$ two-dimensional (2D) hole array drilled in a noble-metal membrane [18] is an emblematic phenomenon in plasmonics. Since ten years, this phenomenon has enabled many theoretical and experimental works [1,18-29] to understand the physical origin of the funneling and squeezing effects at resonance and to exploit them for various applications [1,22-28]. By rigorously solving Maxwell's equations, fully vectorial brute-force calculations [14-16] can reproduce all the salient features of the EOT with high accuracy. However, they only provide an indirect physical insight into the phenomenon, and consequently they weakly guide our intuition in finding design recipes for further engineering plasmonic devices. Recently, these deficiencies have been partly overcome by the introduction of a SPP model [20] that provides a microscopic analysis of the EOT. In contrast with classical grating approaches that emphasize collective properties of the fully periodic system [19,30-32], the model relies on the individual SPPs that are launched in between the holes and that are scattered by the nearby holes to build up the resonant funneling. In essence, this model is similar to the initial model developed by Fano, when he revisited Rayleigh's arguments to explain Wood's anomalies [33] of reflection gratings, by suggesting that a surface mode with a parallel momentum greater than the free space momentum be involved in the energy transport between adjacent grooves to build up a resonance [34]. Although the SPP model [20] well explains and predicts many salient features of the EOT, it provides qualitative predictions that cannot be used with confidence for an accurate analysis. The reason originates from the fact that the SPP model, originally introduced to identify the actual role of SPPs in the EOT, intentionally assumes that only SPPs are responsible for the surface electromagnetic interaction between the holes of the array, therefore neglecting (like in Fano's interpretation) the contribution of field components other than the SPP. This supplementary field component has been identified and analyzed in recent works [21,35-38]; because its field is approximately inversely proportional to the square root of the distance $x$ 
from the line scatterer (at least for $x<10 \lambda[21,36]$ ), it will be consistently called a quasi-cylindrical wave (quasi-CW) hereafter. It has been shown that the SPP and quasi-CW are equally excited at visible frequencies, and that the SPP is weakly excited and the quasi-CW dominates at far-infrared and longer wavelengths $[20,21,36]$.

In this work, we propose what we believe to be a new microscopic model (Section 3), which takes into account both the SPPs and the quasi-CWs. The new model is based on a recently established new formalism [39] (summarized in Section 2 for the sake of completeness), in which the two surface waves, the SPP and the quasi-CW, are simultaneously excited with a fixed relative proportion and thus act as a whole entity that we call a hybrid wave (HW). The HW model drastically enlarges the spectral range of validity of the microscopic SPP model and improves our understanding of the EOT (Section 4). Conclusions are summarized in Section 5.

\section{HYBRID WAVES}

In the SPP model [20], the quasi-CWs are neglected and the SPP scattering events at every individual onedimensional (1D) hole chain are coherently gathered to build up the transmission through the $2 \mathrm{D}$ hole array. The SPP model well predicts all the salient features of the EOT, such as the resonance wavelength and the presence of an antiresonance; however it only approximately predicts the magnitude of the transmission peak in the visible and near-infrared bands, and is largely inaccurate at far-infrared and longer wavelengths, due to the weak excitation of SPPs that are expelled from the metal into the dielectric region.

Introducing the scattering of quasi-CWs into the SPP model is expected to overcome the deficiency of the classical SPP model and to provide a comprehensive overview of the EOT over a broad spectral range. The difficulty lies in the more complex essence of the quasi-CW, compared with the SPP. The SPP is a normal mode and can be treated conveniently by the well-established normal-mode theory (like using the mode scattering coefficients and the related reciprocity) $[40,41]$, while the quasi-CW is not a normal mode.

This difficulty has been recently overcome, thanks to a new HW formalism [39] that allows us to handle the scattering of the quasi-CW in a way formally identical to the classical way we handle the SPP scattering. It is not the aim of this section to present the HW formalism in detail since this has been done in [39]. We rather intend to summarize its main aspects so that a potential reader may easily understand how it is used in the next section to derive analytical expressions for the EOT.

The HW formalism, which takes into account multiple scattering effects such as cross-conversions from SPPs to quasi-CWs and vice versa [42], derives from the finding that for any 2D (invariant along a single $y$-direction) sub- $\lambda$ object on a noble-metal surface under any illumination of transverse-magnetic (TM) polarization (magnetic vector along the $y$-direction), the scattered field is always the same, except for a proportionality factor; it is the HW. Indeed, this finding only holds for sub- $\lambda$ objects, provided that the transverse size of the object is small enough com- pared to the wavelength. A detailed justification of this property can be found in [39]. The HW is thus defined as the radiation of a TM-polarized magnetic line source on the metal surface (at the position of the object). For the HW that is excited by a line source at $x=z=0$ and that propagates in the positive $x$-direction, its magnetic field $H_{\mathrm{HW}}^{+}(x, z)$ on the metal surface $(z=0)$ is $[21,36]$

$$
\begin{aligned}
H_{\mathrm{HW}}^{+}(x, 0)= & H_{\mathrm{SP}}^{+}(x, 0)+H_{\mathrm{CW}}^{+}(x, 0)=\exp \left(i k_{\mathrm{SP}} x\right) \\
& +\left(2 \pi \frac{k_{\mathrm{SP}}^{2}}{k_{0}^{2}} \frac{\sqrt{\varepsilon_{d} \varepsilon_{m}}}{\varepsilon_{m}-\varepsilon_{d}}\right)^{-1}\left(I_{m}+I_{d}\right),
\end{aligned}
$$

if $x>0$ and is zero if $x<0$, where

$$
I_{m}=\exp (-i \pi / 4) \frac{\varepsilon_{d}}{\varepsilon_{m}-\varepsilon_{d}} \int_{0}^{+\infty} \frac{\exp \left(i k_{0} x \sqrt{\varepsilon_{m}+i t}\right) \sqrt{t}}{\left[1-\left(\varepsilon_{m}+i t\right) k_{0}^{2} / k_{\mathrm{SP}}^{2}\right] \sqrt{\varepsilon_{m}+i t}} d t,
$$

and $I_{d}$ can be obtained from $I_{m}$ with $\varepsilon_{d}$ and $\varepsilon_{m}$ exchanged, $\varepsilon_{d}$ and $\varepsilon_{m}$ being the relative permittivities of the dielectric and metallic materials (the $\varepsilon_{m}$ values for gold are taken from the tabulated data in [43] for the following calculations), and $k_{\mathrm{SP}}=k_{0}\left[\varepsilon_{d} \varepsilon_{m} /\left(\varepsilon_{d}+\varepsilon_{m}\right)\right]^{1 / 2}$ being the in-plane SPP propagation constant. $I_{m}+I_{d}$ can be calculated by numerical integration and asymptotically admits a cylindrical-wave behavior of $x^{-1 / 2} \exp \left(i k_{0} \varepsilon_{d}{ }^{1 / 2} x\right)$ in the vicinity of the object $\left(x \ll\left|\varepsilon_{m}\right| \lambda\right)$ [36]. For the HW that propagates in the negative $x$-direction, its magnetic field is given by $H_{\mathrm{HW}}^{-}(x, 0)=H_{\mathrm{HW}}^{+}(-x, 0)$ due to the symmetry. The HW expression of Eq. (1) originates from the Greenfunction calculation of the radiation of a Dirac line-source on a metallic interface. The solution encompasses two contributions: a SPP field (formally identified as a pole contribution), $H_{\mathrm{SP}}^{+}(x, 0)=\exp \left(i k_{\mathrm{SP}} x\right)$, and the quasi-CW field (formally given by a contour integral in the complex plane); see details in [21,36]. Equation (1) shows that the relative proportion of the two contributions is fixed and only depends on $\varepsilon_{d}$ and $\varepsilon_{m}$. On this basis, as shown in [39], it is possible to attach scattering coefficients to the $\mathrm{HW}$, although generally scattering coefficients can be defined only for normal modes [40,41]. Actually, these coefficients are simple since they can be formally identified to SPP scattering coefficients [39].

\section{MICROSCOPIC HYBRID-WAVE MODEL}

Hereafter, we consider a gold membrane in air pierced by a fully periodic $2 \mathrm{D}$ hole array $(\operatorname{period} a$ ) illuminated by a plane wave of TM polarization (magnetic vector along $y$-axis) as shown in Fig. 1(d). Under the assumption that the field transmission through the perforated membrane is mainly mediated by the fundamental Bloch mode of the hole array, the zeroth-order transmission coefficient $t_{F}\left(k_{x}\right)$ can be expressed as a classical Fabry-Perot equation $[19,32,20]$,

$$
t_{F}\left(k_{x}\right)=\frac{t_{A}^{2}\left(k_{x}\right) \exp \left(i k_{0} n d\right)}{1-r_{A}^{2}\left(k_{x}\right) \exp \left(i 2 k_{0} n d\right)},
$$

where $t_{A}\left(k_{x}\right), r_{A}\left(k_{x}\right)$, and $n$ are, respectively, the transmission coefficient, the reflection coefficient, and the complex effective index of the fundamental Bloch mode [see Fig. $1(\mathrm{~d})$ ], with $k_{x}$ being the $x$-component of the wave vector of 


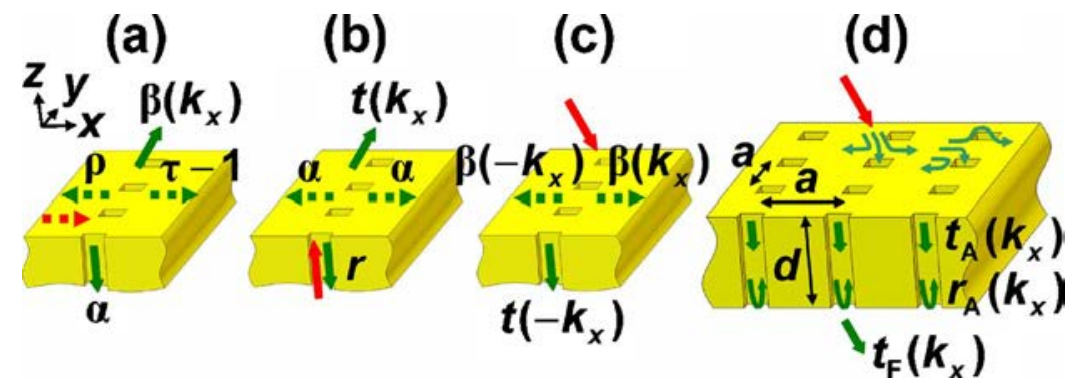

Fig. 1. (Color online) Elementary HW scattering events (a)-(c) for building up the EOT phenomenon (d). (a)-(c) HW scattering coefficients at a 1D hole chain under illumination (a) by a HW, (b) by the fundamental mode of the chain, and (c) by a TM-polarized plane wave. This defines six scattering coefficients, $\rho, \tau, \alpha, \beta\left(k_{x}\right), r$, and $t\left(k_{x}\right)$, with $k_{x}$ being the $x$-component of the wave vector of the incident or scattered plane waves. (d) Modal scattering coefficients used in the classical Fabry-Perot equation of the EOT. The arrows on the surface in the chain and in free space denote HWs, fundamental chain modes, and plane waves, respectively. The arrows denoting incident and scattered waves are in red and in green, respectively.

the incident plane wave, and $d$ is the membrane thickness. Before deriving analytical expressions for $t_{A}\left(k_{x}\right)$ and $r_{A}\left(k_{x}\right)$ in Subsections 3.B and 3.C, we now consider the elementary scattering coefficients associated with the scattering of HWs by a $1 \mathrm{D}$ hole chain.

\section{A. HW Scattering Coefficients}

The main contribution of the HW model is to derive analytical expressions for $t_{A}\left(k_{x}\right)$ and $r_{A}\left(k_{x}\right)$. For that purpose, like in [20], the $2 \mathrm{D}$ hole array is treated as a periodic ensemble of infinite-depth 1D hole chains [Figs. 1(a)-1(c)], with a sub- $\lambda$ period $a$ in the $y$-direction. By virtue of the analogy between SPP and HW scattering coefficients [39], we can define the HW transmission and reflection coefficients, $\tau-1$ and $\rho$ [Fig. 1(a)], and the HW excitation coefficients, $\beta\left(k_{x}\right)$ and $\alpha$, under illumination of the chain by an incident plane wave [Fig. 1(c)] and by the fundamental chain Bloch mode [Fig. 1(b)]. All these HW scattering coefficients can be related to the corresponding SPP ones [39]: thus $\rho$ and $\tau$ can be seen as the reflection and transmission coefficients of the SPP, and $\beta\left(k_{x}\right)$ and $\alpha$ are also the SPP scattering coefficients from an incident plane wave and from the fundamental chain mode to launched SPPs. Thus, due to the reciprocity theorem [41], $\beta\left(k_{x}\right)$ and $\alpha$ are also the scattering coefficients from an incident SPP or HW to a scattered plane wave or to the fundamental chain mode [Fig. 1(a)]. To build up the HW model, we need also to define a reflection coefficient $r$ of the fundamental chain mode [Fig. 1(b)] and a transmission coefficient $t\left(k_{x}\right)$ from the incident fundamental chain mode to the scattered plane wave with a parallel momentum $k_{x}$ [Fig. 1(b)] and reciprocally [Fig. 1(c)].

All these SPP scattering coefficients, $\rho, \tau, \alpha, \beta\left(k_{x}\right), r$, and $t\left(k_{x}\right)$, have been calculated in [20] using an aperiodicFourier modal method (a-FMM) [44]. The a-FMM is a fully vectorial frequency-domain modal method, which is a generalization of the well-developed rigorous coupled wave analysis (RCWA) [16] by including perfectly matched layers to handle the outgoing wave conditions in aperiodic structures.

\section{B. Nonperiodic Array of 1D Hole Chains}

To derive the master equations of the HW model, let us start with a very general case in which a finite set of identical 1D hole chains are arrayed nonperiodically in the $x$-direction and are illuminated by a TM-polarized plane wave at oblique incidence (Fig. 2). According to the main assumption of the HW formalism (Section 2), a set of elementary HWs are launched by every hole chain of the array illuminated by the incident plane wave [Fig. 1(c)], and these HWs further excite new HWs by scattering on the neighboring chains [Fig. 1(a)]. Thus the total near field at the metal surface $(z=0)$ can be written as a superposition of $2 N \mathrm{HWs}$ that originate from the $N$ chains,

$$
H_{y}(x, z)=\sum_{n=1}^{N}\left[P_{n} H_{\mathrm{HW}}^{+}\left(x-x_{n}, z\right)+Q_{n} H_{\mathrm{HW}}^{-}\left(x-x_{n}, z\right)\right]
$$

where we use $P_{n}$ and $Q_{n}$ to, respectively, denote the unknown coefficients of the right-going and the left-going HWs that originate from the $n$th chain located at $x=x_{n}$ $(n=1,2, \ldots, N)$. Referring to the HW scattering coefficients defined in Fig. 1, a set of coupled-HW equations at the $n$th chain can be written as

$$
\begin{aligned}
P_{n}= & W_{n} \beta\left(k_{x}\right)+(\tau-1) \sum_{m=0}^{n-1} P_{m} H_{\mathrm{HW}}^{+}\left(x_{n}-x_{m}, 0\right) \\
& +\rho \sum_{m=n+1}^{N+1} Q_{m} H_{\mathrm{HW}}^{-}\left(x_{n}-x_{m}, 0\right),
\end{aligned}
$$

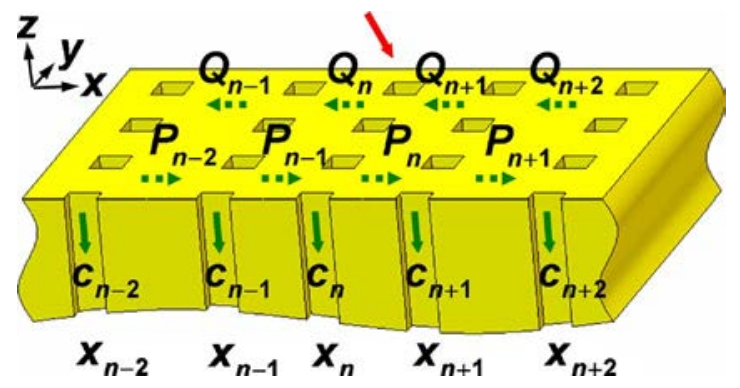

Fig. 2. (Color online) Coupled-wave coefficients of a nonperiodic array of 1D hole chains illuminated by a TM-polarized plane wave at oblique incidence. The notation of arrows follows that in Fig. 1. $P_{n}, Q_{n}$, and $c_{n}$ denote the coefficients of the right-going $\mathrm{HW}$, the left-going HW, and the down-going fundamental chain mode that originate from the $n$th chain at $x=x_{n}(n=1,2, \ldots, N)$. 


$$
\begin{aligned}
Q_{n}= & W_{n} \beta\left(-k_{x}\right)+\rho \sum_{m=0}^{n-1} P_{m} H_{\mathrm{HW}}^{+}\left(x_{n}-x_{m}, 0\right)+(\tau \\
& \text { - 1) } \sum_{m=n+1}^{N+1} Q_{m} H_{\mathrm{HW}}^{-}\left(x_{n}-x_{m}, 0\right),
\end{aligned}
$$

where $W_{n}=\exp \left(i k_{x} x_{n}\right)$ is the phase shift of the incident plane wave at the $n$th chain, with $k_{x}$ being the $x$-component of the wave vector of the incident plane wave, and $H_{\mathrm{HW}}^{ \pm}(x, 0)$ is given by Eq. (1). Equations (4) are intuitive. For Eq. (4a), the three terms represent the excitation of right-going HWs at the $n$th chain by the incident plane wave [Fig. 1(c)], by all the right-going HWs that impinge from the left side of the $n$th chain $(m<n)$ [Fig. 1(a)], or by all the left-going HWs that impinge from the right side of the $n$th chain $(m>n)$. Equation (4b) represents the excitation of left-going HWs at the $n$th chain and can be similarly understood. Combined with the boundary conditions $P_{0}=Q_{N+1}=0$, implying that no $\mathrm{HW}$ is sent from the two outer sides of the chain array, Eqs. (4) form a set of $2 N$ linear equations with $2 N$ unknowns, the $P_{n}$ and $Q_{n}$. This linear system is easily solved for $P_{n}$ and $Q_{n}$ through a matrix inversion for instance. Then the excitation coefficient $c_{n}$ of the down-going fundamental mode in the $n$th chain is determined by

$$
\begin{aligned}
c_{n}= & W_{n} t\left(-k_{x}\right)+\alpha \sum_{m=0}^{n-1} P_{m} H_{\mathrm{HW}}^{+}\left(x_{n}-x_{m}\right)+\alpha \sum_{m=n+1}^{N+1} Q_{m} H_{\mathrm{HW}}^{-}\left(x_{n}\right. \\
& \left.-x_{m}\right)
\end{aligned}
$$

a sum of three terms that are easily understood.

To implement the model, we first calculate the SPP elementary scattering coefficients $\rho, \tau, \alpha, \beta\left(k_{x}\right)$, and $t\left(k_{x}\right)$ defined in Fig. 1 for an individual hole chain. Then we solve Eqs. (4) and use Eq. (5) to determine wave coefficients $P_{n}$, $Q_{n}$, and $c_{n}$, from which we can reproduce both near- and far-field properties. The computational load for implementing the model mainly consists of the fully vectorial a-FMM computation of the SPP elementary scattering coefficients, since in comparison, the computation load required for solving Eqs. (4) through a matrix inversion is negligible. Note that for fully vectorial numerical approaches that treat the aperiodic chain array as a whole entity, they are extremely demanding in computational sources and become unavailable in fact when the number of chains exceeds a few tens, while the computation amount associated with the model always remains at an accessible level since only the elementary unit structure (the individual hole chain) needs to be treated with fully vectorial methods when calculating the SPP scattering coefficients.

\section{Periodic 2D Hole Array}

For an infinite periodic array of 1D hole chains (fully periodic $2 \mathrm{D}$ hole array that supports EOT), we have $N \rightarrow \infty$, $x_{n}=n a$, and $W_{n}=w^{n}$, with $w=\exp \left(i k_{x} a\right)$ being the phase shift of the incident plane wave accumulated in a single period $a$. In addition, according to the Floquet theorem, we have

$$
P_{n}=w P_{n-1}, \quad Q_{n}=w Q_{n-1}, \quad c_{n}=w c_{n-1} .
$$

Rewriting Eqs. (6) as $P_{n}=w^{n} P_{0}, Q_{n}=w^{n} Q_{0}, c_{n}=w^{n} c_{0}$ and inserting them into Eqs. (4) and (5), we can obtain analytical expressions for the unknowns (no need to numerically invert a matrix for fully periodic systems). Now Eqs. (4) and (5) become

$$
\begin{gathered}
P_{0}=\beta\left(k_{x}\right)+(\tau-1) \Sigma H_{\mathrm{HW}}^{+} P_{0}+\rho \Sigma H_{\mathrm{HW}}^{-} Q_{0}, \\
Q_{0}=\beta\left(-k_{x}\right)+\rho \Sigma H_{\mathrm{HW}}^{+} P_{0}+(\tau-1) \Sigma H_{\mathrm{HW}}^{-} Q_{0}, \\
c_{0}=t\left(-k_{x}\right)+\alpha \Sigma H_{\mathrm{HW}}^{+} P_{0}+\alpha \Sigma H_{\mathrm{HW}}^{-} Q_{0},
\end{gathered}
$$

where $\Sigma H_{\mathrm{HW}}^{ \pm}=\Sigma_{n=1}^{\infty} w^{\mp n} H_{\mathrm{HW}}^{+}(n a, 0)$ represents two infinite summations of the HW fields originating at multiples of the period with a phase shift $w . \Sigma H_{\mathrm{HW}}^{ \pm}$can be rewritten as $\Sigma H_{\mathrm{HW}}^{ \pm}=\Sigma H_{\mathrm{SP}}^{ \pm}+\Sigma H_{\mathrm{CW}}^{ \pm}$, with $\Sigma H_{\mathrm{SP}}^{ \pm}=1 /\left(w^{ \pm 1} u^{-1}-1\right)$ representing the SPP contribution $\left[u=\exp \left(i k_{\mathrm{SP}} a\right)\right.$ being the phase shift of the SPP experienced in one period], and with $\Sigma H_{\mathrm{CW}}^{ \pm}=\sum_{n=1}^{\infty} w^{\mp n} H_{\mathrm{CW}}^{+}(n a, 0)$ representing a quasi-CW contribution. The convergence of the infinite summation $\Sigma H_{\mathrm{CW}}^{ \pm}$is ensured by the asymptotic damping for a large $x$ : $\left|H_{\mathrm{CW}}^{+}(x, 0)\right|$ scales as $x^{-3 / 2}$ in the far zone $x \gg\left|\varepsilon_{m}\right| \lambda[36-38]$. The numerical evaluation of $\Sigma H_{\mathrm{CW}}^{ \pm}$can benefit from the analytical expression of Eq. (1). Because the exponential factor in the integrand of $I_{m}$ is almost zero, $I_{m}$ is much smaller than $I_{d}$ and can be neglected. Then performing the infinite sum over the quasi-CW, Eq. (1) becomes

$$
H_{\Sigma, \mathrm{CW}}^{ \pm} \approx\left(2 \pi \frac{k_{\mathrm{SP}}^{2}}{k_{0}^{2}} \frac{\sqrt{\varepsilon_{d} \varepsilon_{m}}}{\varepsilon_{m}-\varepsilon_{d}}\right)^{-1} \exp (-i \pi / 4) \frac{\varepsilon_{m}}{\varepsilon_{d}-\varepsilon_{m}} \int_{0}^{+\infty} \frac{\sqrt{t} /\left\{\exp \left[i a\left( \pm k_{x}-k_{0} \sqrt{\varepsilon_{d}+i t}\right)\right]-1\right\}}{\left[1-\left(\varepsilon_{d}+i t\right) k_{0}^{2} / k_{\mathrm{SP}}^{2}\right] \sqrt{\varepsilon_{d}+i t}} d t
$$

which can be easily calculated through numerical integration. Solving $P_{0}$ and $Q_{0}$ analytically from Eqs. (7a) and (7b), we can get

$$
P_{0}=\frac{\beta\left(k_{x}\right)\left[\left(1 / \Sigma H_{\mathrm{HW}}^{-}+1\right)-\tau\right] / \Sigma H_{\mathrm{HW}}^{+}+\beta\left(-k_{x}\right) \rho / \Sigma H_{\mathrm{HW}}^{+}}{\left[\left(1 / \Sigma H_{\mathrm{HW}}^{+}+1\right)-\tau\right]\left[\left(1 / \Sigma H_{\mathrm{HW}}^{-}+1\right)-\tau\right]-\rho^{2}},
$$

$$
Q_{0}=\frac{\beta\left(-k_{x}\right)\left[\left(1 / \Sigma H_{\mathrm{HW}}^{+}+1\right)-\tau\right] / \Sigma H_{\mathrm{HW}}^{-}+\beta\left(k_{x}\right) \rho / \Sigma H_{\mathrm{HW}}^{-}}{\left[\left(1 / \Sigma H_{\mathrm{HW}}^{+}+1\right)-\tau\right]\left[\left(1 / \Sigma H_{\mathrm{HW}}^{-}+1\right)-\tau\right]-\rho^{2}}
$$

Inserting the expressions of $P_{0}$ and $Q_{0}$ into Eq. (7c), we obtain $c_{0}$, which is nothing else but the transmission coefficient $t_{A}\left(k_{x}\right)$ from the incident plane wave to the fundamental Bloch mode of the periodic $2 \mathrm{D}$ hole array, since the 
latter is composed of a coherent superposition of the fundamental Bloch modes in all chains. The final result for $t_{A}\left(k_{x}\right)$ is

$$
t_{A}\left(k_{x}\right)=t\left(-k_{x}\right)+\alpha \frac{\beta\left(k_{x}\right)\left[\left(1 / \Sigma H_{\mathrm{HW}}^{-}+1\right)-(\tau-\rho)\right]+\beta\left(-k_{x}\right)\left[\left(1 / \Sigma H_{\mathrm{HW}}^{+}+1\right)-(\tau-\rho)\right]}{\left[\left(1 / \Sigma H_{\mathrm{HW}}^{+}+1\right)-\tau\right]\left[\left(1 / \Sigma H_{\mathrm{HW}}^{-}+1\right)-\tau\right]-\rho^{2}} .
$$

Strictly following the same procedure, we easily derive an analytical expression of the reflection coefficient $r_{A}\left(k_{x}\right)$ of the fundamental hole-array Bloch mode. The result is just the right side of Eq. (9a) with $\beta\left(k_{x}\right)$ and $\beta\left(-k_{x}\right)$ replaced by $\alpha$ and with $t\left(k_{x}\right)$ replaced by $r$,

$$
r_{A}\left(k_{x}\right)=r+\alpha^{2} \frac{\left[\left(1 / \Sigma H_{\mathrm{HW}}^{-}+1\right)-(\tau-\rho)\right]+\left[\left(1 / \Sigma H_{\mathrm{HW}}^{+}+1\right)-(\tau-\rho)\right]}{\left[\left(1 / \Sigma H_{\mathrm{HW}}^{+}+1\right)-\tau\right]\left[\left(1 / \Sigma H_{\mathrm{HW}}^{-}+1\right)-\tau\right]-\rho^{2}} .
$$

Equations (9) retain all the features of the SPP model [20] and contain it as a special case without any quasi-CW $\left(\Sigma H_{\mathrm{CW}}^{ \pm}=0\right)$. Under normal illumination $\left(k_{x}=0\right)$, Eqs. (9) take a simpler form,

$$
\begin{aligned}
& t_{\mathbf{A}}\left(k_{x}=0\right)=t+\frac{2 \alpha \beta}{\left(1 / \Sigma H_{\mathrm{HW}}+1\right)-(\rho+\tau)}, \\
& r_{\mathbf{A}}\left(k_{x}=0\right)=r+\frac{2 \alpha^{2}}{\left(1 / \Sigma H_{\mathrm{HW}}+1\right)-(\rho+\tau)},
\end{aligned}
$$

where $\quad t=t\left(k_{x}=0\right), \quad \beta=\beta\left(k_{x}=0\right), \quad$ and $\quad \Sigma H_{\mathrm{HW}}$ $=\Sigma_{n=1}^{\infty} H_{\mathrm{HW}}^{+}(n a, 0)=\Sigma H_{\mathrm{SP}}+\Sigma H_{\mathrm{CW}}$, with $\Sigma H_{\mathrm{SP}}=1 /\left(u^{-1}-1\right)$ and $\Sigma H_{\mathrm{CW}}=\Sigma_{n=1}^{\infty} H_{\mathrm{CW}}^{+}($na, 0$)$.

\section{Perfect Conductor Case}

At very low (terahertz to microwave) frequencies for which metals act as perfect conductors, the implementation of the HW model deserves special attention, since now SPPs cannot be excited efficiently and only quasiCWs contribute $[20,21,17,45]$. As metals approach perfect conductors $\left(\varepsilon_{m} \rightarrow \infty\right)$, we can observe that $\rho \rightarrow 0, \tau \rightarrow 1, \alpha$ $\rightarrow 0, \beta\left(k_{x}\right) \rightarrow 0$, and $\Sigma H_{\mathrm{HW}}^{ \pm} \rightarrow \infty$. Then we find that the second fraction terms in the model equations (9) become an indefinite form of type "0/0." Now the problem arises on how to calculate the limit of such indefinite forms. The solution relies on theoretical knowledge of scaling laws for the scattering coefficients as $\varepsilon_{m}$ increases. It has been shown that $[36,46]$

$$
\begin{aligned}
\rho= & \varepsilon_{m}^{-1 / 2} \rho^{(\mathrm{PC})}, \quad \tau-1=\varepsilon_{m}^{-1 / 2}\left(\tau^{(\mathrm{PC})}-1\right), \\
& \alpha=\varepsilon_{m}^{-1 / 4} \alpha^{(\mathrm{PC})}, \quad \beta\left(k_{x}\right)=\varepsilon_{m}^{-1 / 4} \beta^{(\mathrm{PC})}\left(k_{x}\right),
\end{aligned}
$$

where the quantities with a superscript "PC" tend to constants as $\varepsilon_{m} \rightarrow \infty$, and this notation is consistently used in the following. Since the field scattered by a hole chain for an incident fundamental chain mode or plane wave approaches constant as metals approach perfect conductors, we have

$$
r=r^{(\mathrm{PC})}, \quad t\left(k_{x}\right)=t^{(\mathrm{PC})}\left(k_{x}\right) .
$$

Moreover, since $I_{m} \rightarrow 0, I_{d} \rightarrow$ const, and $k_{\mathrm{SP}} \rightarrow k_{0}$ as $\varepsilon_{m} \rightarrow \infty$ [see Eq. (1)], we get

$$
\Sigma H_{\mathrm{CW}}^{ \pm}=\varepsilon_{m}^{1 / 2} \Sigma H_{\mathrm{CW}}^{ \pm(\mathrm{PC})}, \quad \Sigma H_{\mathrm{SP}}^{ \pm}=\Sigma H_{\mathrm{SP}}^{ \pm(\mathrm{PC})} \text {. }
$$

Equations (11c) confirm the anticipated fact [20,21,36] that the contribution of SPPs, relatively to that of quasi$\mathrm{CWs}$, vanishes as $\varepsilon_{m} \rightarrow \infty$. Inserting Eqs. (11) into the model equations (9) and letting $\varepsilon_{m} \rightarrow \infty$, we can obtain for perfect conductors

$$
\begin{gathered}
t_{A}\left(k_{x}\right)=t^{(\mathrm{PC})}\left(-k_{x}\right)+\alpha^{(\mathrm{PC})} \frac{\beta^{(\mathrm{PC})}\left(k_{x}\right)\left[\left(1 / \Sigma H_{\mathrm{CW}}^{-(\mathrm{PC})}+1\right)-\left(\tau^{(\mathrm{PC})}-\rho^{(\mathrm{PC})}\right)\right]+\beta^{(\mathrm{PC})}\left(-k_{x}\right)\left[\left(1 / \Sigma H_{\mathrm{CW}}^{+(\mathrm{PC})}+1\right)-\left(\tau^{(\mathrm{PC})}-\rho^{(\mathrm{PC})}\right)\right]}{\left[\left(1 / \Sigma H_{\mathrm{CW}}^{+(\mathrm{PC})}+1\right)-\tau^{(\mathrm{PC})}\right]\left[\left(1 / \Sigma H_{\mathrm{CW}}^{-(\mathrm{PC})}+1\right)-\tau^{(\mathrm{PC})}\right]-\left(\rho^{(\mathrm{PC})}\right)^{2}}, \\
r_{A}\left(k_{x}\right)=r^{(\mathrm{PC})}+\left(\alpha^{(\mathrm{PC})}\right)^{2} \frac{\left[\left(1 / \Sigma H_{\mathrm{CW}}^{-(\mathrm{PC})}+1\right)-\left(\tau^{(\mathrm{PC})}-\rho^{(\mathrm{PC})}\right)\right]+\left[\left(1 / \Sigma H_{\mathrm{CW}}^{+(\mathrm{PC})}+1\right)-\left(\tau^{(\mathrm{PC})}-\rho^{(\mathrm{PC})}\right)\right]}{\left[\left(1 / \Sigma H_{\mathrm{CW}}^{+(\mathrm{PC})}+1\right)-\tau^{(\mathrm{PC})}\right]\left[\left(1 / \Sigma H_{\mathrm{CW}}^{-(\mathrm{PC})}+1\right)-\tau^{(\mathrm{PC})}\right]-\left(\rho^{(\mathrm{PC})}\right)^{2}} .
\end{gathered}
$$

Equations (12) represent a pure quasi-CW model of EOT for perfect conductors, in which the contribution of SPPs vanishes as expected. The form of Eqs. (12) is identical to that of Eqs. (9) except that the scattering coefficients correspond to a perfectly conducting case. Under normal incidence, Eqs. (12) reduce to 


$$
\begin{aligned}
& t_{A}\left(k_{x}=0\right)=t^{(\mathrm{PC})}+\frac{2 \alpha^{(\mathrm{PC})} \beta^{(\mathrm{PC})}}{\left(1 / \Sigma H_{\mathrm{CW}}^{(\mathrm{PC})}+1\right)-\left(\rho^{(\mathrm{PC})}+\tau^{(\mathrm{PC})}\right)}, \\
& r_{A}\left(k_{x}=0\right)=r^{(\mathrm{PC})}+\frac{2\left(\alpha^{(\mathrm{PC})}\right)^{2}}{\left(1 / \Sigma H_{\mathrm{CW}}^{(\mathrm{PC})}+1\right)-\left(\rho^{(\mathrm{PC})}+\tau^{(\mathrm{PC})}\right)},
\end{aligned}
$$

where $t^{(\mathrm{PC})}=t^{(\mathrm{PC})}\left(k_{x}=0\right), \quad \beta^{(\mathrm{PC})}=\beta^{(\mathrm{PC})}\left(k_{x}=0\right)$, and $\Sigma H_{\mathrm{CW}}^{(\mathrm{PC})}$ $=\Sigma H_{\mathrm{CW}}^{ \pm(\mathrm{PC})}\left(k_{x}=0\right)$.

Theoretically, the calculation of the normalized scattering coefficients $\rho^{(\mathrm{PC})}, \tau^{(\mathrm{PC})}, \alpha^{(\mathrm{PC})}, \beta^{(\mathrm{PC})}\left(k_{x}\right), r^{(\mathrm{PC})}$, and $t^{(\mathrm{PC})}$ $\times\left(k_{x}\right)$ can be made very accurate by considering very large values of $\varepsilon_{m}$. However, in practice, dealing with very large $\varepsilon_{m}$ 's is not an easy task in general [47]. In particular, since the "SPP" mode spreads far away in the dielectric region at long wavelengths (the mode is no longer well confined on the interface), the calculation of their scattering coefficients is problematical with the a-FMM. In addition, near the resonance wavelength, $t_{A}\left(k_{x}\right)$ and $r_{A}\left(k_{x}\right)$ become extremely sensitive to the values of these scattering coefficients (especially to the values of $\rho^{(\mathrm{PC})}$ and $\tau^{(\mathrm{PC})}$ that appear in the denominator); thus a very high precision is required for the calculation. Due to these dif- ficulties, we rather prefer fitting the fully vectorial data of EOT spectra obtained for infinite conductivity with the model equations to determine the scattering coefficients. We use the fully vectorial method in [48] to compute the spectra. As will be shown in Figs. 4(c) and 4(d) below, the predictions of the model show perfect agreement with the fully vectorial data.

\section{PREDICTIONS OF THE MICROSCOPIC HYBRID-WAVE MODEL}

A. Comparison between Fully Vectorial Data and Model Predictions

To evidence the validity and the high precision of the HW model, we have compared the model predictions with fully vectorial RCWA $[16,49]$ computational data and with the predictions of the pure-SPP model [20]. Figure 3 shows the comparison for different incident angles and for the near-infrared band. All the data are obtained for a gold membrane in air perforated by a fully periodic $2 \mathrm{D}$ hole array. Figure 3(a) shows the zeroth-order transmittance $T$ $=\left|t_{F}\left(k_{x}\right)\right|^{2}$ for various incident angles $\theta$ and wavelengths $\lambda$ (note that $k_{x}=2 \pi \lambda^{-1} \sin \theta$ ). Figures 2(b) and 2(c) show the zeroth-order transmittance and reflectance spectra for two incident angles: $\theta=0^{\circ}$ and $5^{\circ}$. The deviation between the RCWA data and the HW-model predictions is almost imperceptible. (a)

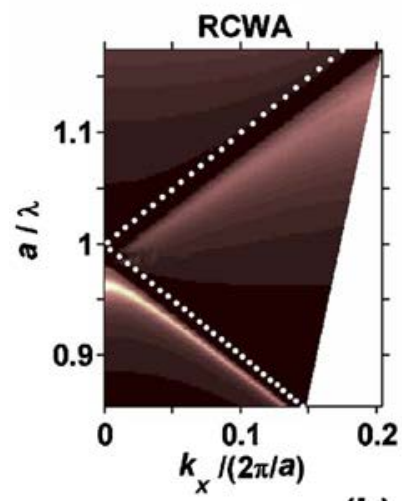

(b)

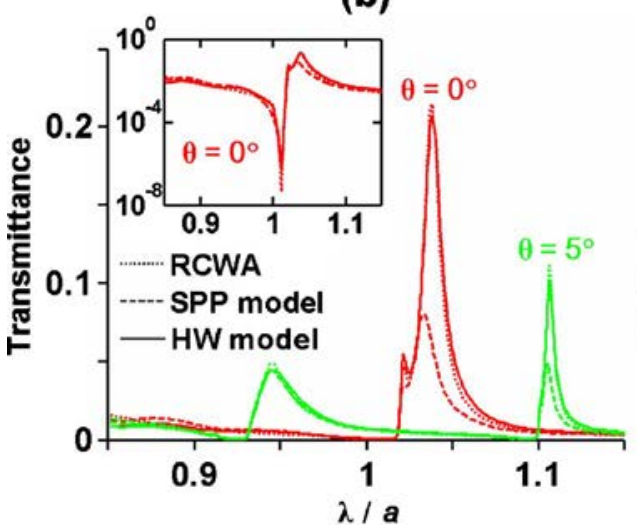

SPP model

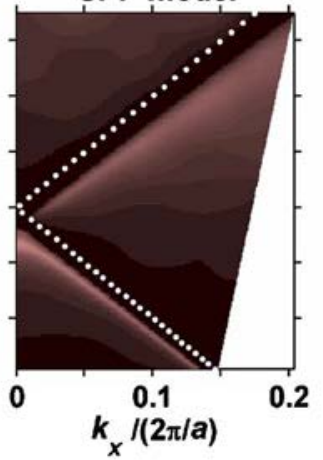

HW model

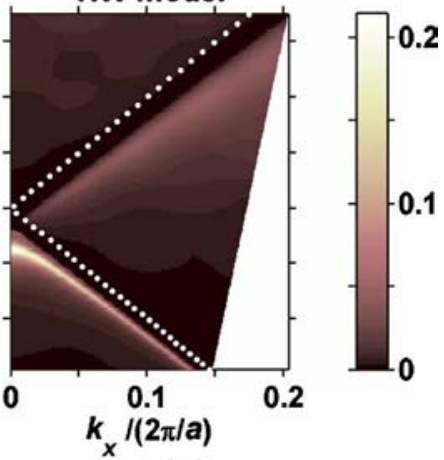

(c)

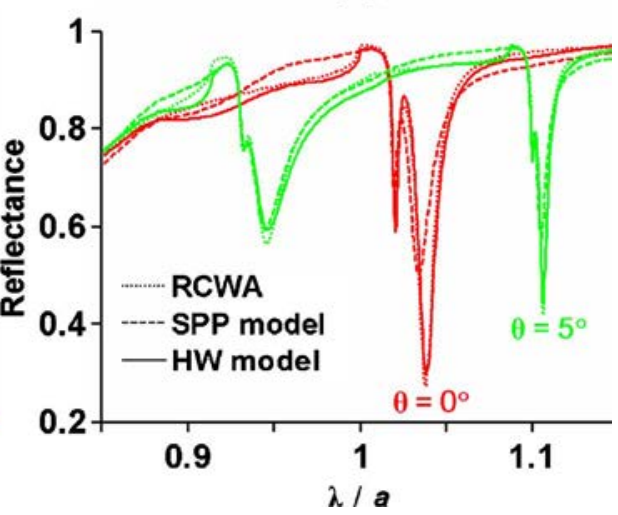

Fig. 3. (Color online) Comparison between the RCWA data and the model predictions for different incident angles $\theta$ and for the nearinfrared band. All the data are obtained for a gold membrane in air perforated by a periodic 2D array of square holes; the period is $a$ $=0.94 \mu \mathrm{m}$, the hole side length is $D=0.266 \mu \mathrm{m}$, and the membrane thickness is $d=0.2 \mu \mathrm{m}$. (a) Zeroth-order transmittance obtained with the RCWA (left), the SPP model (middle), and the HW model (right). The dotted-white lines represent the air light lines, at which a diffraction order propagates parallel to the metal surface. (b),(c) Zeroth-order transmittance and reflectance spectra for two incident angles $\theta=0^{\circ}$ (red) and $5^{\circ}$ (green), obtained with the RCWA (dotted), the SPP model (dashed), and the HW model (solid). The inset in (b) shows the transmittance in a logarithmic scale and evidences the existence of a deep transmission minimum. 
Additional comparisons performed at visible, thermalinfrared, and low frequencies for which metals act as perfect conductors are shown in Fig. 4. Again the HW model exhibits perfect agreement with the fully vectorial data at all frequencies that are below the gold plasma frequency in the blue; in comparison the SPP model becomes less and less precise at long wavelengths. This can be explained by the fact that the excitation of SPPs becomes weaker at longer wavelengths and even vanishes for perfect conductors, while the excitation of quasi-CWs remains almost constant at all these frequencies $[21,36]$.

\section{B. Phase-Matching Condition}

Compared with the pure-SPP model [20], the new model that incorporates both SPPs and quasi-CWs provides a richer and more quantitative insight into the EOT. For small holes that support only evanescent modes, the enhanced transmission is attributed to the very large values of $t_{A}$ and $r_{A}[19,32,20]$, which originate from the zero of the denominator in the model equations (9) or (10). It has been shown that for small holes that scatter little energy, $\rho \approx 0, \tau \approx 1$, and $\arg (\tau+\rho) \approx \arg (\tau)$ is a very small positive number [20]. So the denominator in Eqs. (10) under normal incidence can be made very close to zero, provided that $\Sigma H_{\mathrm{HW}}$ has a large modulus and satisfies a phasematching condition,

$$
\arg \left(1 / \Sigma H_{\mathrm{HW}}+1\right)=\arg (\tau+\rho) \approx \arg (\tau) \bmod 2 \pi .
$$

Equation (14) contains the phase-matching condition given by the SPP model as a special case consisting of neglecting the quasi-CWs $\left(\Sigma H_{\mathrm{CW}}=0\right)$. Since the in-plane propagation constants of the quasi-CW and of the SPP are both quite close to the free-space propagation constant $k_{0}=2 \pi / \lambda$ [36], very large value of $\left|\Sigma H_{\mathrm{HW}}\right|$ is anticipated for $\lambda \approx a$, for which the HW fields scattered by all individual chains add up constructively. This analysis is confirmed in Fig. 5. The peak wavelength of the transmittance $\left|t_{A}\right|^{2}$ is exactly predicted by the phase-matching condition (14), while the peak wavelength predicted by the SPP model is blueshifted. The enhanced agreement can be explained by the slightly lower phase and modulus of $1 / \Sigma H_{\mathrm{HW}}+1$ compared with $1 / \Sigma H_{\mathrm{SP}}+1=\exp \left(-i k_{\mathrm{SP}} a\right)$, near the resonance wavelength. Note that if the SPP (a)

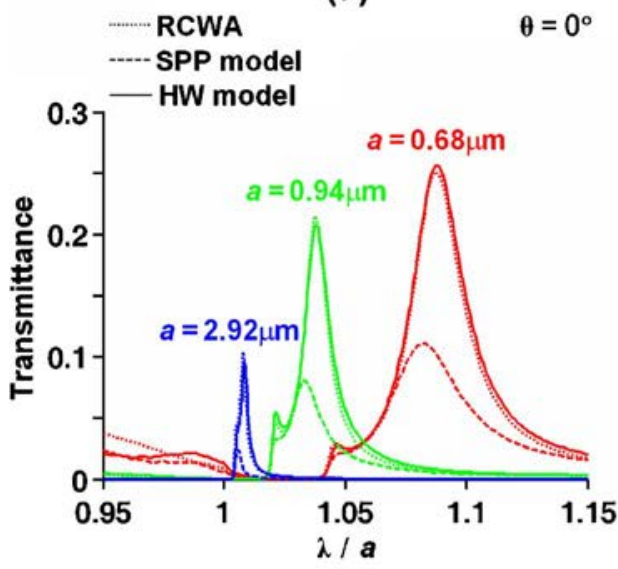

(c)

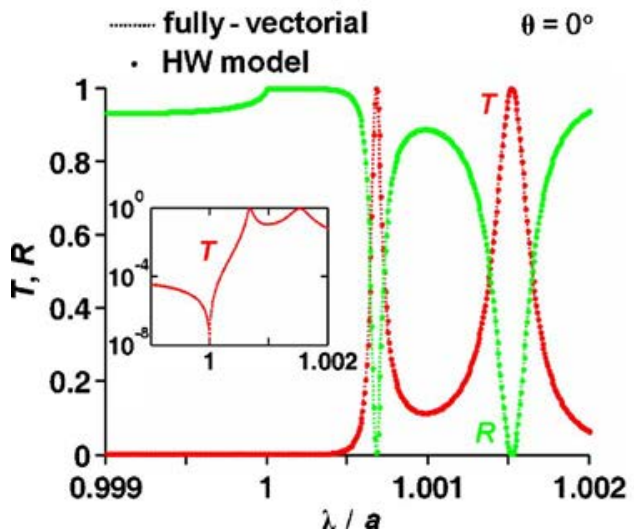

(b)

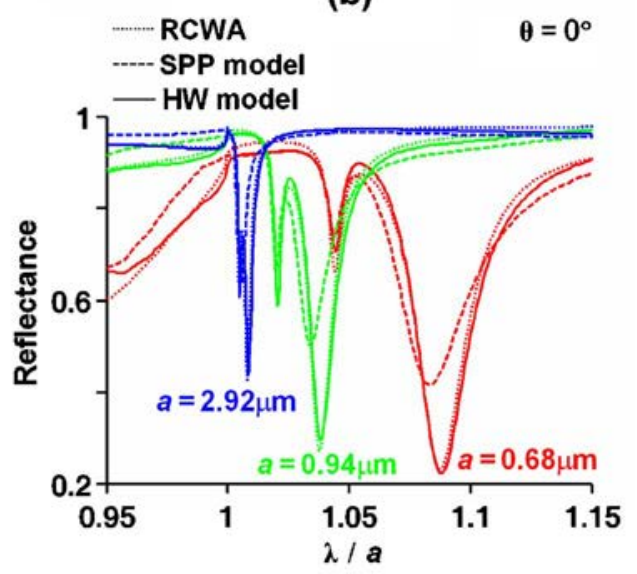

(d)

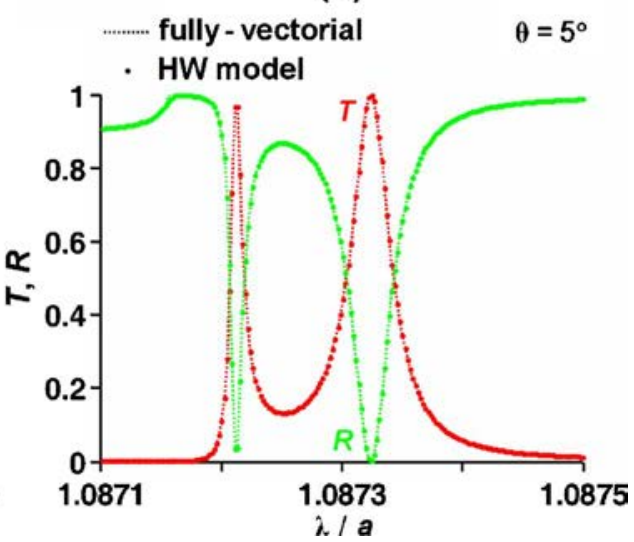

Fig. 4. (Color online) Comparison between the fully vectorial data and the model predictions for various wavelength ranges. All the data are obtained for a gold membrane in air perforated by a periodic $2 \mathrm{D}$ array of square holes; the hole side length is $D / a=0.28$, and the membrane thickness is $d / a=0.21$, with $a$ being the grating period. (a),(b) Zeroth-order transmittance and reflectance spectra under normal incidence, which are obtained with the RCWA (dotted), the SPP model (dashed), and the HW model (solid) and are shown in the visible (red, $a=0.68 \mu \mathrm{m}$ ), the near-infrared (green, $a=0.94 \mu \mathrm{m}$ ), and the thermal-infrared (blue, $a=2.92 \mu \mathrm{m}$ ) bands. (c),(d) Perfect conductor results under normal and oblique incidence $\left(\theta=5^{\circ}\right)$, which show the zeroth-order transmittance $T$ (red) and reflectance $R$ (green) spectra for very low frequencies. The fully vectorial data are shown with dotted curves and the HW-model predictions with dot marks. The inset in (c) shows $T$ in a logarithmic scale. 


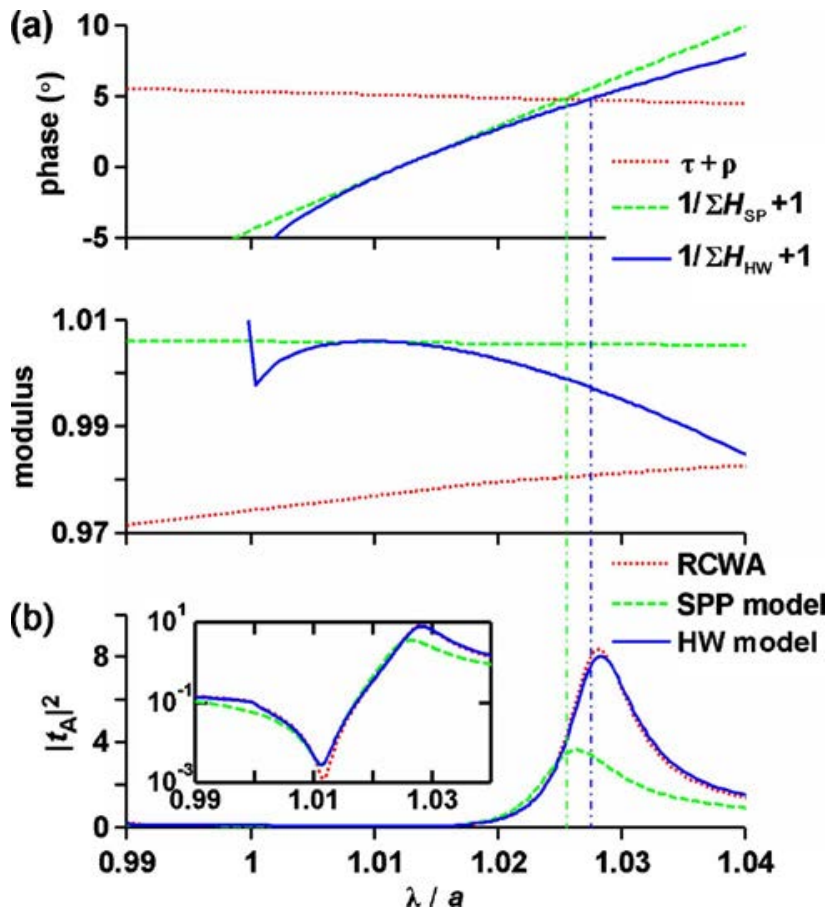

Fig. 5. (Color online) Phase-matching condition under normal incidence. (a) The phase (upper) and the modulus (lower) of $\tau$ $+\rho$ (dotted-red lines), $1 / \Sigma H_{\mathrm{SP}}+1=\exp \left(-i k_{\mathrm{SP}} a\right)$ (dashed-green lines), and $1 / \Sigma H_{\mathrm{HW}}+1$ (solid-blue lines). (b) Transmittance $\left|t_{A}\right|^{2}$ obtained with the RCWA (dotted-red lines), the pure SPP model (dashed-green lines), and the HW model (solid-blue lines). The inset shows $\left|t_{A}\right|^{2}$ in a logarithmic scale. The phase-matching condition, which corresponds to the two intersections in (a) for predicting the peak wavelength of $\left|t_{A}\right|^{2}$, is labeled by the left and right dashed-dotted vertical lines for the SPP model and for the HW model, respectively.

mode matches the incident light through the reciprocal grating momentum, i.e., if $\lambda / a=\operatorname{Re}\left(k_{\mathrm{SP}}\right) / k_{0} \approx 1.01$ at normal incidence, the transmittance exhibits a deep dip that is predicted by both models [inset in Fig. 5(b)]; there $1 / \Sigma H_{\mathrm{HW}}+1 \approx 1 / \Sigma H_{\mathrm{SP}}+1$ as shown in Fig. 5(a). The model is also valid for oblique incidence [see Eqs. (9)]. The resonance occurs for $\left( \pm k_{x}-k_{0}\right) a \approx 0 \bmod 2 \pi$ [corresponding to the two white-dotted light lines in Fig. 3(a)] and is exactly located by the phase-matching conditions of $\arg \left(1 / \Sigma H_{\mathrm{HW}}^{ \pm}+1\right) \approx \arg (\tau) \bmod 2 \pi \quad$ [corresponding to the two transmission branches in Fig. 3(a)].

Although out of the main stream of the present paper, it is worth mentioning that the $\mathrm{HW}$ model is again quantitative for arrays of large holes, annular holes [50] or slits [28], which support a single propagative aperture mode. This is because the Fabry-Perot equation (2) remains valid when the aperture supports a single propagative mode $[28,50]$. However, in such cases, the physics of the enhanced transmission is quite different, since now the crucial scattering process shown in Fig. 1(a) is no longer energy conservative: except for very narrow apertures, $|\tau|$ is considerably smaller than 1 because a nonnegligible fraction of the incident SPP energy is carried away by the propagative aperture mode.

\section{CONCLUSION}

We have presented a comprehensive model of the extraordinary optical transmission (EOT) through periodic sub- $\lambda$ metallic hole arrays. The model relies on a new formalism [39], which assumes that the field scattered by sub- $\lambda$ objects on metallic surfaces is always composed (for a given frequency) of a fixed proportion of a surface plasmon polariton (SPP) and of a quasi-cylindrical wave (quasi-CW) [Eq. (1)]. The total field is called a hybrid wave (HW). Thanks to a formal analogy with the classical scattering coefficients of SPPs, we have defined scattering coefficients [Figs. 1(a)-1(c)] for this wave, which is not a normal mode. By writing down coupled-wave equations for the set of HWs generated at every aperture, we have obtained analytical expressions [Eqs. (9)-(13)] for the EOT [Fig. 1(d)]. Compared with the pure-SPP model proposed in [20], the HW model provides much more accurate predictions over a much broader spectral range, from the visible to the far-infrared and microwave regions of the spectrum (Figs. 3 and 4). This arises from the fact that the present model additionally takes into account the quasiCWs that dominate SPPs at far-infrared and longer wavelengths $[21,36]$. The model clarifies the exact roles of the SPP and of the quasi-CW in forming the EOT phenomenon [Fig. 5 and Eq. (14)] and clarifies the impact of the frequency-dependent metal conductivity in the EOT $[18,51,52]$, an issue that has largely puzzled initial interpretations $[53,54,30]$. We believe that the model is valid in general for explaining many Wood-type anomalies $[33,34]$ on metallic surfaces, and that it can facilitate the design of various surface-resonance-based devices [1,22-28,51,52] operating from visible to microwave frequencies.

\section{ACKNOWLEDGMENTS}

The authors thank Jean Paul Hugonin. H. Liu acknowledges financial support from the National Natural Science Foundation of China (NSFC) (No. 10804057), from the Cultivation Fund of the Key Scientific and Technical Innovation Project, Ministry of Education of China (No. 708021), from the Program for New Century Excellent Talents in University (No. NCET-08-0289), and from the 973 Project (No. 2007CB307001).

\section{REFERENCES AND NOTES}

1. C. Genet and T. W. Ebbesen, "Light in tiny holes," Nature 445, 39-46 (2007).

2. M. E. Stewart, C. R. Anderton, L. B. Thompson, J. Maria, S. K. Gray, J. A. Rogers, and R. G. Nuzzo, "Nanostructured plasmonic sensors," Chem. Rev. (Washington, D.C.) 108, 494-521 (2008).

3. S. P. Burgos, R. de Waele, A. Polman, and H. A. Atwater, "A single-layer wide-angle negative-index metamaterial at visible frequencies," Nature Mater. 9, 407-412 (2010).

4. H. F. Schouten, N. Kuzmin, G. Dubois, T. D. Visser, G. Gbur, P. F. A. Alkemade, H. Blok, G. W. 't Hooft, D. Lenstra, and E. R. Eliel, "Plasmon-assisted two-slit transmission: Young's experiment revisited,” Phys. Rev. Lett. 94, 053901 (2005).

5. C. H. Gan, G. Gbur, and T. D. Visser, "Surface plasmons modulate the spatial coherence of light in Young's interference experiment," Phys. Rev. Lett. 98, 043908 (2007).

6. L. Cai, G. Y. Li, Z. H. Wang, and A. S. Xu, "Interference and horizontal Fabry-Perot resonance on extraordinary transmission through a metallic nanoslit surrounded by grooves," Opt. Lett. 35, 127-129 (2010).

7. L. Yin, V. K. Vlasko-Vlasov, J. Pearson, J. M. Hiller, J. Hua, U. Welp, D. E. Brown, and C. W. Kimball, "Subwavelength focusing and guiding of surface plasmons," Nano Lett. 5, 1399-1402 (2005). 
8. F. López-Tejeira, S. G. Rodrigo, L. Martín-Moreno, F. J. García-Vidal, E. Devaux, T. W. Ebbesen, J. R. Krenn, I. P. Radko, S. I. Bozhevolnyi, M. U. González, J. C. Weeber, and A. Dereux, "Efficient unidirectional nanoslit couplers for surface plasmons," Nat. Phys. 3, 324-328 (2007).

9. I. I. Smolyaninov, D. L. Mazzoni, and C. C. Davis, "Imaging of surface plasmon scattering by lithographically created individual surface defects," Phys. Rev. Lett. 77, 3877-3880 (1996).

10. C. Genet, M. P. van Exter, and J. P. Woerdman, "Huygens description of resonance phenomena in subwavelength hole arrays,” J. Opt. Soc. Am. A 22, 998-1002 (2005).

11. O. T. A. Janssen, H. P. Urbach, and G. W. 't Hooft, "On the phase of plasmons excited by slits in a metal film," Opt. Express 14, 11823-11832 (2006).

12. G. Y. Li, L. Cai, F. Xiao, Y. J. Pei, and A. S. Xu, "A quantitative theory and the generalized Bragg condition for surface plasmon Bragg reflectors," Opt. Express 18, 1048710499 (2010)

13. Q. Q. Gan, Y. K. Gao, Q. Wang, L. Zhu, and F. Bartoli, "Surface plasmon waves generated by nanogrooves through spectral interference,” Phys. Rev. B 81, 085443 (2010).

14. R. Petit, Electromagnetic Theory of Gratings (SpringerVerlag, 1980)

15. A. Taflove, Computational Electrodynamics: The FiniteDifference Time-Domain Method (Artech House, 1995).

16. M. G. Moharam, D. A. Pommet, E. B. Grann, and T. K. Gaylord, "Stable implementation of the rigorous coupled-wave analysis for surface-relief gratings: enhanced transmittance matrix approach," J. Opt. Soc. Am. A 12, 1077-1086 (1995).

17. B. Wang and P. Lalanne, "How many surface plasmons are locally excited on the ridges of metallic lamellar gratings?" Appl. Phys. Lett. 96, 051115 (2010).

18. T. W. Ebbesen, H. J. Lezec, H. F. Ghaemi, T. Thio, and P. A. Wolff, "Extraordinary optical transmission through subwavelength hole arrays," Nature 391, 667-669 (1998).

19. L. Martín-Moreno, F. J. García-Vidal, H. J. Lezec, K. M. Pellerin, T. Thio, J. B. Pendry, and T. W. Ebbesen, "Theory of extraordinary optical transmission through subwavelength hole arrays," Phys. Rev. Lett. 86, 1114-1117 (2001).

20. H. T. Liu and P. Lalanne, "Microscopic theory of the extraordinary optical transmission," Nature 452, 728-731 (2008).

21. P. Lalanne and J. P. Hugonin, "Interaction between optical nano-objects at metallo-dielectric interfaces," Nat. Phys. 2, $551-556$ (2006)

22. M. M. Alkaisi, R. J. Blaikie, S. J. McNab, R. Cheung, and D. R. S. Cumming, "Sub-diffraction-limited patterning using evanescent near-field optical lithography," Appl. Phys. Lett. 75, 3560-3562 (1999).

23. X. G. Luo and T. Ishihara, "Sub-100-nm photolithography based on plasmon resonance," Jpn. J. Appl. Phys., Part 1 43, 4017-4021 (2004).

24. S. Collin, F. Pardo, and J. L. Pelouard, "Resonant-cavityenhanced subwavelength metal-semiconductor-metal photodetector," Appl. Phys. Lett. 83, 1521-1523 (2003).

25. C. Liu, V. Kamaev, and Z. V. Vardeny, "Efficiency enhancement of an organic light emitting diode with a cathode forming two-dimensional periodic hole array," Appl. Phys. Lett. 86, 143501 (2005).

26. M. Diwekar, S. Blair, and M. Davis, "Increased light gathering capacity of sub-wavelength conical metallic apertures," J. Nanophotonics 4, 043504 (2010).

27. G. A. Zheng, X. Q. Cui, and C. H. Yang, "Surface-waveenabled darkfield aperture for background suppression during weak signal detection," Proc. Natl. Acad. Sci. U.S.A. 107, 9043-9048 (2010).

28. S. Collin, G. Vincent, R. Haidar, N. Bardou, S. Rommeluere, and J. L. Pelouard, "Nearly perfect Fano transmission resonances through nanoslits drilled in a metallic membrane," Phys. Rev. Lett. 104, 027401 (2010).

29. F. J. García de Abajo, "Colloquium: Light scattering by particle and hole arrays," Rev. Mod. Phys. 79, 1267-1290 (2007).

30. J. B. Pendry, L. Martin-Moreno, and J. F. Garcia-Vidal, "Mimicking surface plasmons with structured surfaces,"
Science 305, 847-848 (2004).

31. F. J. García de Abajo and J. J. Saenz, "Electromagnetic surface modes in structured perfect-conductor surfaces," Phys. Rev. Lett. 95, 233901 (2005).

32. P. Lalanne, J. C. Rodier, and J. P. Hugonin, "Surface plasmons of metallic surfaces perforated by nanohole arrays," J. Opt. A, Pure Appl. Opt. 7, 422-426 (2005).

33. R. W. Wood, "On a remarkable case of uneven distribution of light in a diffraction grating spectrum," Philos. Mag. 4 396-402 (1902).

34. U. Fano, "The theory of anomalous diffraction gratings and of quasi-stationary waves on metallic surfaces (Sommerfeld's waves)," J. Opt. Soc. Am. 31, 213-222 (1941).

35. L. Aigouy, P. Lalanne, J. P. Hugonin, G. Julie, V. Mathet, and M. Mortier, "Near-field analysis of surface waves launched at nano-slit apertures," Phys. Rev. Lett. 98, 153902 (2007).

36. P. Lalanne, J. P. Hugonin, H. T. Liu, and B. Wang, "A microscopic view of the electromagnetic properties of sub- $\lambda$ metallic surfaces," Surf. Sci. Rep. 64, 453-469 (2009).

37. W. Dai and C. M. Soukoulis, "Theoretical analysis of the surface wave along a metal-dielectric interface," Phys. Rev. B 80, 155407 (2009)

38. A. Y. Nikitin, S. G. Rodrigo, F. J. García-Vidal, and L. Martín-Moreno, "In the diffraction shadow: Norton waves versus surface plasmon polaritons in the optical region," New J. Phys. 11, 123020 (2009).

39. H. T. Liu and P. Lalanne, "Light scattering by metallic surfaces with subwavelength patterns," Phys. Rev. B 82, 115418 (2010).

40. Normal modes are defined as waveguide modes that obey an exponential propagation rule $\exp \left(i k_{0} n_{\mathrm{eff}} z\right)$ along the invariant $z$-direction of the waveguide; see details in [41].

41. C. Vassallo, Optical Waveguide Concepts (Elsevier, 1991).

42. X. Y. Yang, H. T. Liu, and P. Lalanne, "Cross-conversion between surface plasmon polaritons and quasi-cylindrical waves," Phys. Rev. Lett. 102, 153903 (2009).

43. E. D. Palik, Handbook of Optical Constants of Solids, Part II (Academic, 1985).

44. E. Silberstein, P. Lalanne, J. P. Hugonin, and Q. Cao, "Use of grating theories in integrated optics," J. Opt. Soc. Am. A 18, 2865-2875 (2001)

45. D. A. Hill and J. R. Wait, "Excitation of the Zenneck surface wave by a vertical aperture," Radio Sci. 13, 969-977 (1978).

46. H. T. Liu, P. Lalanne, X. Y. Yang, and J. P. Hugonin, "Surface plasmon generation by subwavelength isolated objects," IEEE J. Sel. Top. Quantum Electron. 14, 1522-1529 (2008).

47. A. Roberts and R. C. McPhedran, "Power losses in highly conducting lamellar gratings," J. Mod. Opt. 34, 511-538 (1987).

48. C. C. Chen, "Transmission of microwave through perforated flat plates of finite thickness," IEEE Trans. Microwave Theory Tech. MTT-21, 1-6 (1973).

49. J. P. Hugonin and P. Lalanne, Reticolo Software for Grating Analysis (Institut d'Optique, 2005).

50. F. I. Baida, Y. Poujet, J. Salvi, D. Van Labeke, and B. Guizal, "Extraordinary transmission beyond the cut-off through sub- $\lambda$ annular aperture arrays," Opt. Commun. 282, 1463-1466 (2009).

51. J. Gómez Rivas, C. Schotsch, P. Haring Bolivar and H. Kurz, "Enhanced transmission of $\mathrm{THz}$ radiation through subwavelength holes," Phys. Rev. B 68, 201306(R) (2003).

52. Y. H. Ye and J. Y. Zhang, "Middle-infrared transmission enhancement through periodically perforated metal films," Appl. Phys. Lett. 84, 2977-2979 (2004).

53. P. Lalanne, J. P. Hugonin, S. Astilean, M. Palamaru, and K. D. Möller, "One-mode model and Airy-like formulae for onedimensional metallic gratings," J. Opt. A, Pure Appl. Opt. 2, 48-51 (2000).

54. G. Gay, O. Alloschery, B. Viaris de Lesegno, C. O’Dwyer, J. Weiner, and H. J. Lezec, "The optical response of nanostructured surfaces and the composite diffracted evanescent wave model," Nat. Phys. 2, 262-267 (2006). 